\title{
SAR Image Segmentation Using Morphological Attribute Profiles
}

\author{
Markus Boldt ${ }^{\mathrm{a}, \mathrm{b}}$, Antje Thiele ${ }^{\mathrm{a}, \mathrm{b}}$, Karsten Schulz $^{\mathrm{a}}$, Stefan Hinz ${ }^{\mathrm{b}}$ \\ ${ }^{\text {a }}$ Fraunhofer Institute of Optronics, System Technologies and Image Exploitation - \\ $(<$ markus.boldt $>$, <antje.thiele $>$, $<$ karsten.schulz $>) @$ iosb.fraunhofer.de \\ ${ }^{\mathrm{b}}$ Institute of Photogrammetry and Remote Sensing (IPF), Karlsruhe Institute of Technology (KIT) - \\ (<antje.thiele>, <stefan.hinz>)@kit.edu
}

KEY WORDS: Synthetic Aperture Radar, SAR, TerraSAR-X, Object-Based Image Analysis, OBIA, Mathematical Morphology, Attribute Profiles, AP, Differential Attribute Profiles, DAP, segmentation

\begin{abstract}
:
In the last years, the spatial resolution of remote sensing sensors and imagery has continuously improved. Focusing on spaceborne Synthetic Aperture Radar (SAR) sensors, the satellites of the current generation (TerraSAR-X, COSMO-SykMed) are able to acquire images with sub-meter resolution. Indeed, high resolution imagery is visually much better interpretable, but most of the established pixel-based analysis methods have become more or less impracticable since, in high resolution images, self-sufficient objects (vehicle, building) are represented by a large number of pixels. Methods dealing with Object-Based Image Analysis (OBIA) provide help. Objects (segments) are groupings of pixels resulting from image segmentation algorithms based on homogeneity criteria. The image set is represented by image segments, which allows the development of rule-based analysis schemes. For example, segments can be described or categorized by their local neighborhood in a context-based manner.

In this paper, a novel method for the segmentation of high resolution SAR images is presented. It is based on the calculation of morphological differential attribute profiles (DAP) which are analyzed pixel-wise in a region growing procedure. The method distinguishes between heterogeneous and homogeneous image content and delivers a precise segmentation result.
\end{abstract}

\section{INTRODUCTION}

In the last years, the spatial resolution of remote sensing sensors and imagery has continuously improved. Focusing on spaceborne SAR sensors, the satellites of the current generation (TerraSAR-X, COSMO-SykMed) are able to acquire images with sub-meter resolution. As example, TerraSAR-X (TSX) imagery acquired in HS300 mode has a slant pixel spacing of $0.45 \mathrm{~m}$ (range) and $0.86 \mathrm{~m}$ (azimuth). Indeed, high resolution (HR) imagery is visually much better interpretable, but most of the established pixel-based analysis methods have become more or less impracticable since, in HR images, semantically selfsufficient structures (vehicles, buildings) are represented by a large number of pixels. In urban areas, for example, smallscaled building features like single edges are distinguishable in HR SAR imagery [Soergel et al, 2006].

Since objects (segments), in ideal case, represent semantically self-sufficient structures, methods dealing with Object-Based Image Analysis (OBIA) provide help. Segments are groupings of pixels resulting from image segmentation algorithms based on homogeneity criteria. The image set is represented by image segments, which allows the development of rule-based analysis schemes. For example, segments can be described or categorized by their local neighborhood in a context-based manner.

In general, many different segmentation methods exist and can mainly be discriminated between detecting discontinuities (edge detection) and detecting similarities (thresholding, region growing, etc.) [Narkhede, 2013]. Region growing can be explained as process which merges the image pixels due to a pre-selected homogeneity criterion. This criterion defines the similarity between the pixels and can be chosen to consider the intensity values [Narkhede, 2013] of pixels in a neighborhood of a predefined seed point. In the Multiresolution Segmentation method of Definiens software, for example, the region growing criterion consists of spectral (color) and shape heterogeneity which are combined by a fusion value [Lizarazo et al., 2011]. The shape criterion is defined by the parameters compactness and smoothness. Drawback of this method is that the parameters can be manually weighted depending on the specific dataset. Hence, an optimal parameter setting has to be manually investigated in a trial-and-error procedure.

In contrast, the method proposed in this paper works completely unsupervised. It considers the morphological criterion given by Attribute Profiles (APs) as homogeneity criterion. Morphological Attribute Profiles (APs) [Dalla Mura et al., 2010] can be explained as an advancement of Morphological Profiles (MPs) and were originally developed for the analysis of HR optical remote sensing images. APs base on the sequential calculation of attribute openings (closings). In contrast to traditional morphological openings (closings), connected components (CCs) are evaluated using attribute openings (closings) and no structuring element (SE) has to be defined [Boldt et al., 2013]. Furthermore, since many different attributes are useable, attribute openings (closings) establishing APs are more flexible than the SE based MPs. In other words, the specific image content can be modelled more completely using APs. In this study, the concept of APs is transferred to the segmentation of HR SAR amplitude images. For this, the SAR specific attributes Cov (coefficient of variation) and NRCS (Normalized Radar Cross Section) are used and for each image pixel, both a Cov and an NRCS AP vector are calculated. In the region growing procedure, the mean Euclidian distance between the AP vectors is analysed.

As dataset, a TSX (HS300) time series consisting of ten amplitude images covering the airport of Stuttgart (Germany) and surroundings is used. Basis for the presented segmentation approach are two subset images of the time series mean amplitude image containing heterogeneous (urban) and homogeneous (rural) structures. In a final step, the segmentation results are evaluated and compared with segmentations derived by using Definiens Developer software. 
The paper is organized as follows: First, the preprocessing of the dataset is described (Section 2). In Section 3, the DAP concept and the attributes used in this study are explained. Section 4 contains the proposed segmentation method. Afterwards, the results are evaluated (Section 5) and summarized (Section 6).

\section{DATA PREPROCESSING}

A time series of ten TSX HS300 images (November 2013 May 2014) covering Stuttgart (Germany) airport and surroundings is investigated. The scene center of the area is at $48.68^{\circ} / 9.216^{\circ}$ (lat / lon; WGS84 ellipsoid). The coverage is approximately $10 \times 6 \mathrm{~km}$, the pixel spacing is $0.45 \mathrm{~m}$ in range and $0.86 \mathrm{~m}$ in azimuth. The polarization of all images is $\mathrm{HH}$. The scene contains very heterogeneous land cover types. Besides the airport area, urban, suburban and rural areas are imaged. Furthermore, highways and one railway line is contained.

As pre-processing for the segmentation, the amplitude images are co-registered to one master scene (temporarily oldest image). Furthermore, radiometric calibration is performed for each co-registered amplitude image. Afterwards, the temporal multi-look (ML) mean is calculated from the calibrated images. In the end, the ML mean is scaled to 256 gray values [Boldt et al., 2014]. This enables the usage of a morphological alternating sequential filter (ASF) [Soille, 1998] utilizing the area attribute aiming on a denoised segmentation input [Boldt et al., 2014]. The ASF is well suited for noisy images containing bright and dark structures of different sizes [Soille, 1998]. Moreover, the ASF considers CCs of an image. Doing so, it does not distort the original image content.

From the pre-processed ML mean, two subset images are chosen as segmentation input. Both approx. $400 \times 400$ pixelsized test images contain fine urban (heterogeneous) and coarse rural (homogeneous) structures (Figure 2). In order to get an optimal denoised segmentation input in which all relevant structures are preserved, an adaptively ASF filtering is performed (Figure 1). For this, a Coefficient of variation (Cov) image is calculated and homogeneous / heterogeneous regions are detected using the knowledge about the Cov value of an ideal homogeneous region (Section 3.3). In consequence, homogeneous and heterogeneous structures are ASF filtered independently (Figure 1) and merged together afterwards. For heterogeneous structures, an ASF value sequence preserving fine structures is chosen - for homogeneous regions, a coarser sequence of values is applied. The denoised subset images are used below as input for the proposed segmentation method.

\section{MORPHOLOGICAL ANALYSIS}

The concept of APs and their derivatives DAPs (Differential Attribute Profiles) was originally established by [Dalla Mura et al., 2010] and applied exploiting HR optical remote sensing imagery. In [Boldt et al., 2013], DAPs were transferred to HR SAR images aiming on the automatic detection of similar changes. In this study, we utilize this concept for the unsupervised segmentation of HR SAR amplitude images (Section 2).

APs and DAPs principally base on the attribute filtering [Breen et al., 1996] of CCs of an image considering a sequence of values of a predefined attribute.

In the following, APs and DAPs are explained in more detail.

\subsection{Morphological Attribute Profiles (APs)}

Considering one attribute and a sequence of attribute values (e.g. area, $[2,4]$ ), an AP for one input image $I$ is calculated by iterative attribute openings and closings for each attribute value. Referring to the given example, the AP itself is accomplished by stacking the filtered images as follows:

$$
A P_{\text {area }}(I)=\left[\Gamma_{4, c l, C C}^{A}(I) ; \Gamma_{2, c l, C C}^{A}(I) ; I ; \Gamma_{2, o p, C C}^{A}(I) ; \Gamma_{4, o p, C C}^{A}(I)\right],
$$

where $\Gamma_{2, c l, C C}^{A}(I)$ denotes the attribute closing of CCs using the first value (2) of the sequence defined above. The names of the other images are analogically denoted. It can be seen, that in this example, a 1 x 5-sized AP results. In other words: For each pixel of the input image, a $1 \times 5$ sized AP vector is calculated which is the input for the DAP analysis.

\subsection{Differential Morphological Attribute Profiles (DAPs)}

DAPs are the derivatives of APs. The example given above shows the following structure for the DAP:

$$
D A P_{\text {area }}(I)=\left[\Delta_{1} ; \Delta_{2} ; \Delta_{3} ; \Delta_{4}\right] \text {, }
$$

where:

$$
\begin{aligned}
\Delta_{1} & =\Gamma_{4, c l, c C}^{A}(I)-\Gamma_{2, c l, C C}^{A}(I), \quad \Delta_{2}=\Gamma_{2, c l, c C}^{A}(I)-I, \\
\Delta_{3} & =I-\Gamma_{2, o p, c C}^{A}(I), \Delta_{4}=\Gamma_{2, o p, C C}^{A}(I)-\Gamma_{4, o p, C C}^{A}(I) .
\end{aligned}
$$

In the present example, a $1 \times 4$ DAP vector for each pixel of the input image $I$ is derived which contains the contrast between adjacent AP components. These contrast values deliver information about the sensibility of $\mathrm{CCs}$ concerning on the attribute and the value sequence chosen. In detail: A DAP vector of a single pixel shows whether this pixel is removed or preserved during the attribute filtering. This behavior is the characteristic morphological signature of the pixel.

\subsection{Attributes}

In addition to the area attribute, many other different attributes can be considered calculating APs and DAPs. For example, attributes characterizing statistics, shape or radiometry of $\mathrm{CCs}$ can be utilized.

In this study, we use DAPs for the segmentation of SAR images. Hence, the two specific SAR attributes Cov (Coefficient of varation) and NRCS (Normalized Radar Cross Section) are applied. Consequently, Cov and NRCS values are calculated in each CC of the denoised ML mean subset images. For this, the original ML mean subset image (Cov) and the logarithmized ML mean subset image (NRCS) are considered.

The Cov is the ratio of the standard deviation divided by the mean value of a given sample (image pixels). Usually, the Cov image is calculated using a sliding kernel (e.g. $5 \times 5$ sized) on the input amplitude or intensity image. The Cov can be interpreted as a local homogeneity measure. Relatively high Cov values result in regions which appear locally heterogeneous, like urban or forest. In contrast, regions which contain fully-developed Speckle such as low backscattering meadows or fields exhibit a $\mathrm{Cov}$ which is close to the ideal value of homogeneous areas.

Thanks to squaring in the calibration procedure, the calibrated amplitude images used in our study are in detail intensities and exhibit an exponential distributed Speckle. According to [Ulaby, 1982], the ratio of standard deviation to mean (Cov) for an averaged ML intensity image with exponential distribution is given by: 

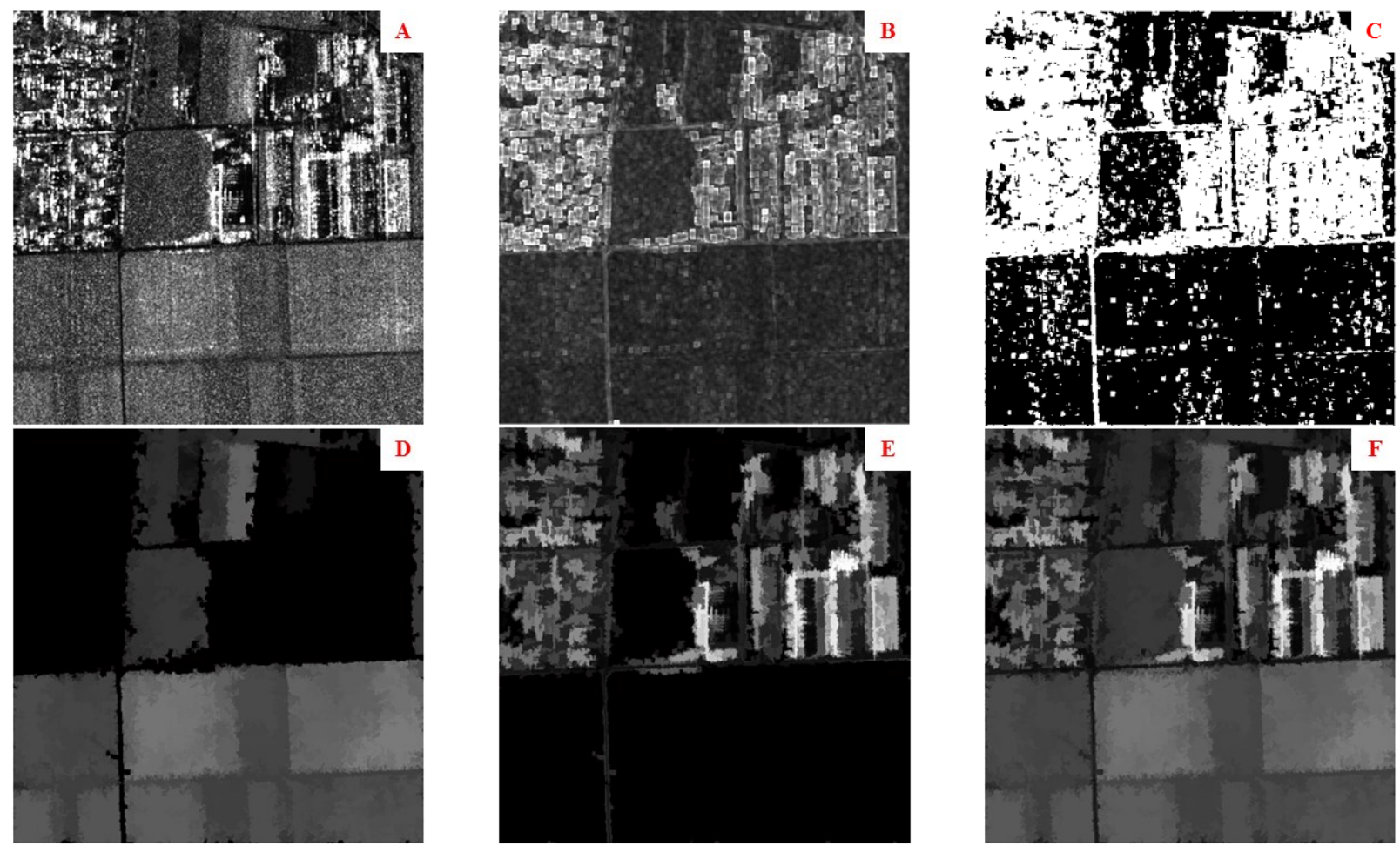

Figure 1: Adaptive ASF denoising: input ML mean subset image (A); Cov 5 x 5 of input image (B); detected heterogeneous (white) and homogeneous (black) regions (C); denoised homogeneous regions (D), denoised heterogeneous regions (E) and combined result (F).

$$
\operatorname{Cov}_{\text {Ideal }, M L, N}=\frac{1}{\sqrt{N}}
$$

where $N$ is the number of independent looks. For $N=10$, the expected $\mathrm{Cov}$ for a homogeneous area with fully developed speckle is approximately 0.32 . This value is used to detect homogeneous structures for the unsupervised adaptive ASF (Section 2) denoising (Figure 1).

Establishing the Cov value sequence for calculating the APs and DAPs, the indiviudal Cov value range for each test site is evaluated. This range is equally divided into ten parts which results in a $1 \times 10$ AP vector and therefore in a $1 \times 9$ DAP vector for each pixel.

The normalized radar cross section NRCS is the radar cross section per unit area of surface. It is often expressed in decibels [dB] by applying the logarithmic function at the natural (normalized) backscatter values [Richards, 2009]. Considering NRCS values, the backscatter of different surface types or even the backscatter of images acquired by different sensors are comparable in a single frame. Since TSX images are used, the noise level can be approximately assumed at $-22 \mathrm{~dB}$ [Schulz et al., 2012] and is chosen as lower boundary for the NRCS value sequence. As upper boundary, $+10 \mathrm{~dB}$ was taken in reference to [Schulz et al., 2012] which is a backscatter value that can typically observed in, for example, industrial areas. Similar to the Cov value sequence, this range is also equally divided into ten values.

\section{SEGMENTATION}

Input for our new segmentation method are the $1 \times 9$ sized DAP Cov and NRCS vectors calculated for each pixel. Doing so, pixel characterizing morphological feature vectors can be considered as homogeneity criterion in a region growing algorithm.

This is done by analyzing the morphological similarity between two neighboring pixels $a$ and $b$ calculating the mean Euclidian distance $\delta$ between their DAP vectors:

$$
\begin{gathered}
\delta_{1}=\left[\sqrt{\left(\sum_{i=1}^{n}\left|D A P_{C o v, a, i}-D A P_{C o v, b, i}\right|^{2}\right)}\right] \\
\delta_{2}=\left[\sqrt{\left(\sum_{i=1}^{n}\left|D A P_{N R C S, a, i}-D A P_{N R C S, b, i}\right|^{2}\right)}\right] \\
\delta=\frac{\delta_{1}+\delta_{2}}{2} .
\end{gathered}
$$

Two pixels in this context are considered as morphologically similar, if $\delta$ is smaller than a threshold $\tau . \tau$ is estimated beforehand by investigating the Euclidian distances between pixels in a detected homogeneous region (Section 2 and Section 3.3). Consequently, $\tau$ is fixed for the particular subset image.

The region growing starts in a randomly chosen seed point of the subset image and tests the similarity to the eight neighboring pixels considering the threshold $\tau$. Initially, the seed pixel itself represents the first (unmerged) segment. If at least one similar pixel is found, it is merged with the seed point resulting in a two pixel sized segment. After merging, new DAP mean vectors (Cov and NRCS) are calculated for the current segment. This step is repeated up to the point that no more pixels are found which are similar to the current segment. Then, a new seed point is chosen from the set of unsegmented pixels and a new segment is generated. The algorithm stops, when all pixels are segmented.

This region growing works completely unsupervised.

For evaluation, the two ASF denoised test sites are also segmented using the OBIA software package Definiens Developer. As algorithm, the Multiresolution Segmentation (MRS) procedure is used, which represents a region growing method that minimizes iteratively the average heterogeneity of pixels / segments [Happ et al., 2010]. The heterogeneity criterion is implemented as being subdivided into a spatial and a 

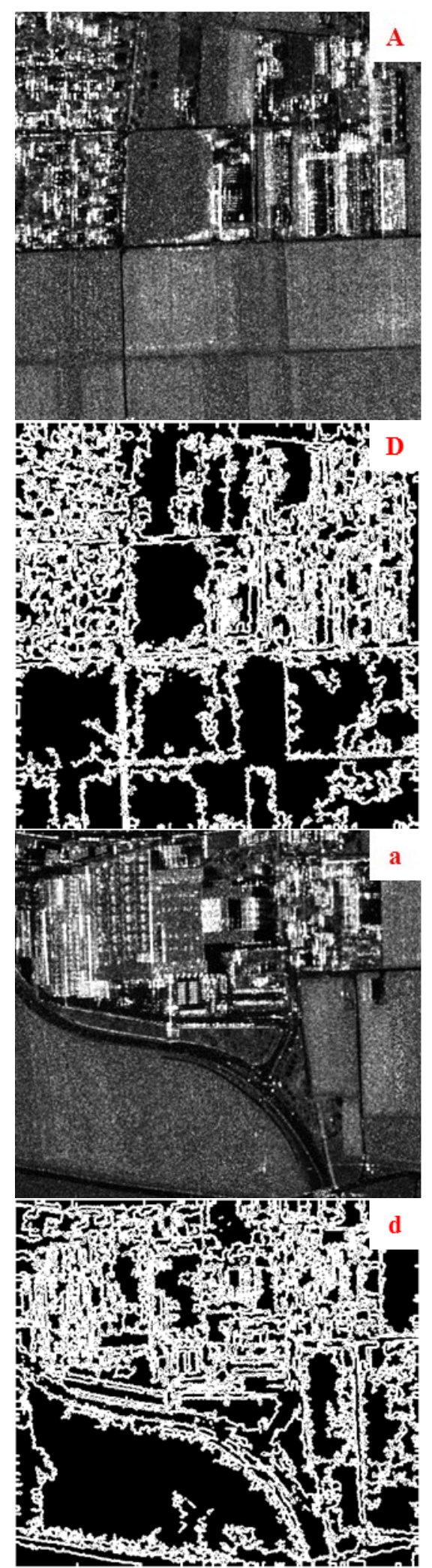
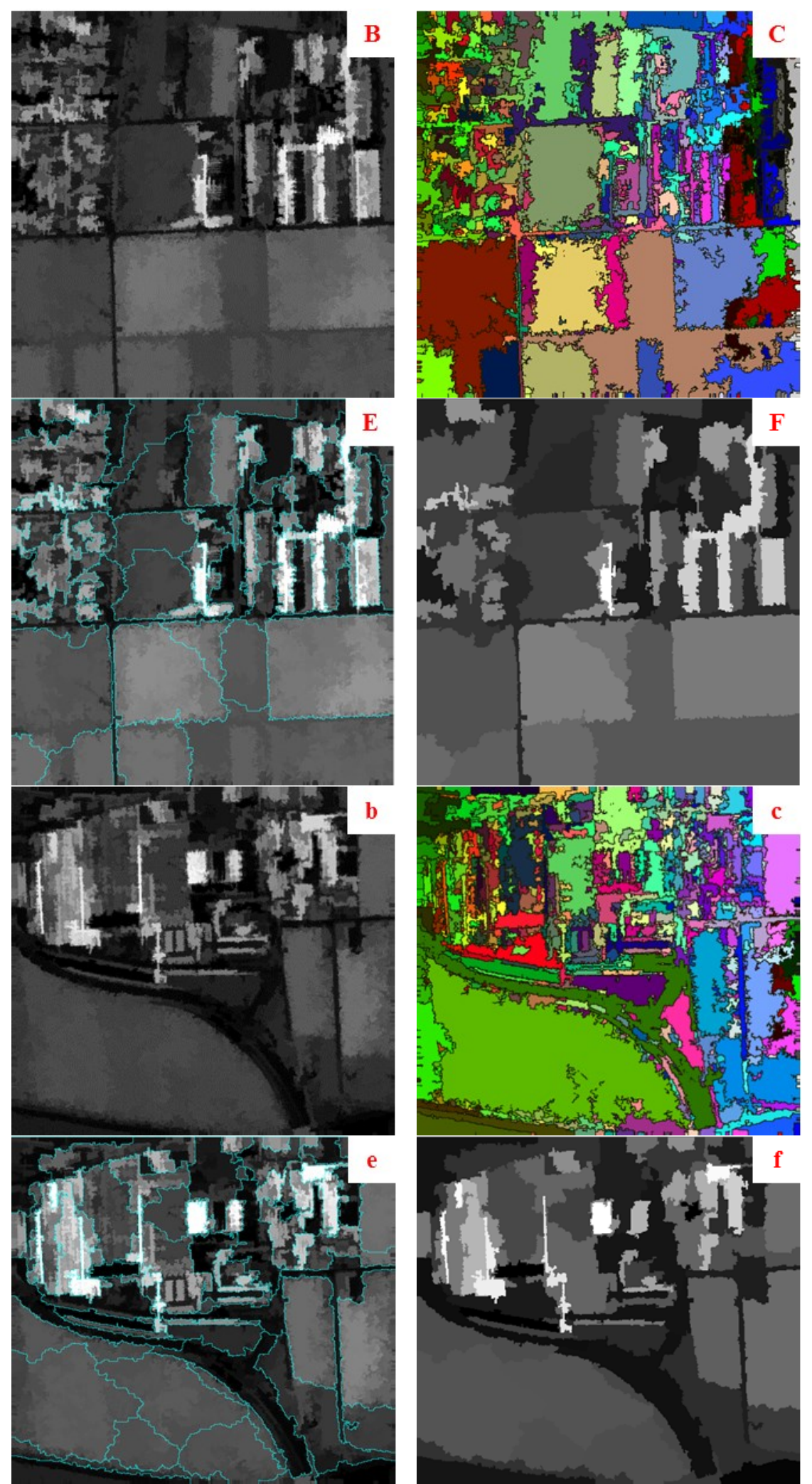

Figure 2: Segmentation results of subset images (A, a); denoised ML mean subset (B, b); color coded segments found by proposed method (C, c); outlines of segments found by proposed method (D, d); outlines of segments found by MRS method (E, e); visualized segment mean values found by MRS method $(F, f)$.

spectral heterogeneity, the spatial heterogeneity is further represented by compactness and smoothness. The weights for the heterogeneity criteria can be set by the operator, and regulate the appearance of the resulting segments.
Moreover, the increase of heterogeneity of segments caused by the region growing is represented by the fusion factor (f) [Happ et al., 2010]. This parameter regulates the merging of similar pixels /segments by being tested against a threshold called Scale Parameter (SP). 
In this study, spatial and spectral heterogeneity weights are set equally to 0.5 . Since homogeneous areas are evaluated in this paper, the coarse segmentation result using a SP of 100 is used as input.

\section{RESULTS AND EVALUATION}

To evaluate the segmentation results of the proposed method, reference segments representing homogeneous areas are selected manually based on the CovAmCoh [Boldt et al., 2012] image. The reference segments are compared with the segments found by the DAP driven segmentation and with the segments detected by the MRS procedure in Definiens Developer. For both test sites, three reference segments were selected (Figure $3)$. These segments are used as binary mask which is intersected with the segmentation results. Due to this fusion, three pixel categories result: reference pixels which are correctly segmented (true positives TP); reference pixels which are not segmented (false negatives FN) and pixels which are segmented but not part of the reference segment (false positives FP) [Passat et al., 2011].

The results of the evaluation are listed in Table 1. It can be seen that the percentage of TP pixels is significantly higher using the DAP driven approach. Moreover, focusing on the two segments ' 3 ', the DAP based approach apparently delivers better results than the MRS segmentation. This is caused by the coarse MRS segmentation used in this evaluation. Since the segments ' 3 ' represent relatively fine homogeneous structures, they are not suitably detected by the MRS segmentation. In the DAP segmentation, those structures are detected, since it does not need any information concerning the size of resulting segments. Additionally, it can be mentioned that the DAP based segmentation method delivers very promising and accurate results.

\section{SUMMARY AND OUTLOOK}

In this study, a new method for unsupervised segmentation of HR time series SAR images is presented. This method is based on Morphological Differential Attribute Profiles (DAPs). As attributes, the SAR specific parameters Coefficient of variation (Cov) and normalized radar cross section (NRCS) are chosen. For each test pixel, $1 \times 9 \mathrm{Cov}$ and NRCS DAP vectors are calculated and used as heterogeneity criterion in a region growing segmentation algorithm.

The results are evaluated considering manually selected reference segments and are compared to another method given by the Multiresolution Segmentation implemented in Definiens Developer software. It is shown that the DAP based segmentation delivers quite accurate results. Thus, it will be used in further studies. These investigations comprise for example the usage of the segments for analyzing the local spatial context of HR time series SAR amplitude images. The proposed method will be part of a change analysis performed on HR time series SAR imagery focusing on the categorization of detected changes.

\section{REFERENCES}

Boldt, M., Thiele, A., Schulz, K., Hinz, S., 2014. Unsupervised Segmentation of HR Time Series SAR Amplitude Imagery Aiming on Context Based Change Categorization. In: Proceedings of the $10^{\text {th }}$ European Conference on Synthetic Aperture Radar EUSAR, Berlin, Germany, pp. 616-619.
Boldt, M., Schulz, K., Thiele, A., Hinz, S., 2013. Using Morphological Differential Attribute Profiles for Change Categorization in High Resolution SAR Images. In: International Archives of the Photogrammetry, Remote Sensing and Spatial Information Sciences, ISPRS Hannover Workshop, Vol. XL-1/W1, pp. 29-34.

Boldt, M., Schulz, K., 2012: Change Detection in Time Series of High Resolution SAR Satellite Images. In: Proceedings of SPIE Vol. 8538, Earth Resources and Environmental Remote Sensing / GIS Applications III, pp. 853806-1 - 853806-9.

Breen, E. J., Jones, R., 1996. Attribute Openings, Thinnings and Granulometries. In: Computer Vision and Image Understanding, 64(3), Article No. 0066, pp. 377 - 389.

Dalla Mura, M., Benediktsson, J. A., Waske, B., Bruzzone, L., 2010. Morphological Attribute Profiles for the Analysis of Very High Resolution Images. In: IEEE Transactions on Geoscience and Remote Sensing, Vol. 48, No. 10, pp. 3747 - 3762.

Happ, N. P., Ferreira, R. S., Bentes, C., Costa, G. A. O. P., Feitosa, R. Q., 2010. Multiresolution Segmentation: A Parallel Approach for High Resolution Image Segmentation in Multicore Architectures. In: International Conference on Geographic Object-Based Image Analysis, ISPRS Vol. XXXVIII-4/C7, 6 p.

Lizarazo, I., Elsner, P., 2011: Segmentation of Remotely Sensed Imagery: Moving from Sharp Objects to Fuzzy Regions, In: Image Segmentation, Publisher: InTech, ISBN: 978-953-307228-9, $550 \mathrm{p}$.

Narkhede, H. P., 2013. Review of Image Segmentation Techniques. In: International Journal of Science and Modern Engineering (IJSME), ISSN: 2319-6386, Vol. 1, pp. 54-61.

Passat, N., Naegel, B., Rousseau, F., Koob, M., Dietemann, J.L. 2011. Interactive segmentation based on component-trees. In: Pattern Recognition 44, 10-11 (2011), 27 p.

Richards, J. A., 2009. Remote Sensing with Imaging Radar. Series: Signals and Communication Technology, Springer Verlag, ISBN: 3642020194, 361 p.

Schulz, K., Boldt, M., Even, M., 2012. Generalization of the CovAmCoh Analysis for the Interpretation of Arbitrary InSAR Images. In: Proceedings of the International Geoscience and Remote Sensing Symposium IGARSS, pp. 7444 - 7447.

Soergel, U., Thoennessen, U., Brenner, A., Stilla, U. 2006. High-resolution SAR data: new opportunities and challenges for the analysis of urban areas. In: IEE Proceedings. Radar, sonar \& navigation 153, No. 3, pp. $294-300$.

Soille, P., 1998. Morphological Image Analysis: Principles and Applications. Springer Verlag Berlin Heidelberg New York, ISBN: 3540429883, $391 \mathrm{p}$.

Ulaby, F. T., Moore, R. K., Fung, A. K., 1982. Microwave Remote Sensing: Active and Passive. Volume II: Radar Remote Sensing and Surface Scattering and Emission Theory. AddisonWesley Publishing Company, Advanced Book Program / World Science Division, Reading, Massachusetts, ISBN: 0-201-10760$0,1064 \mathrm{p}$. 
The International Archives of the Photogrammetry, Remote Sensing and Spatial Information Sciences, Volume XL-3, 2014 ISPRS Technical Commission III Symposium, 5 - 7 September 2014, Zurich, Switzerland
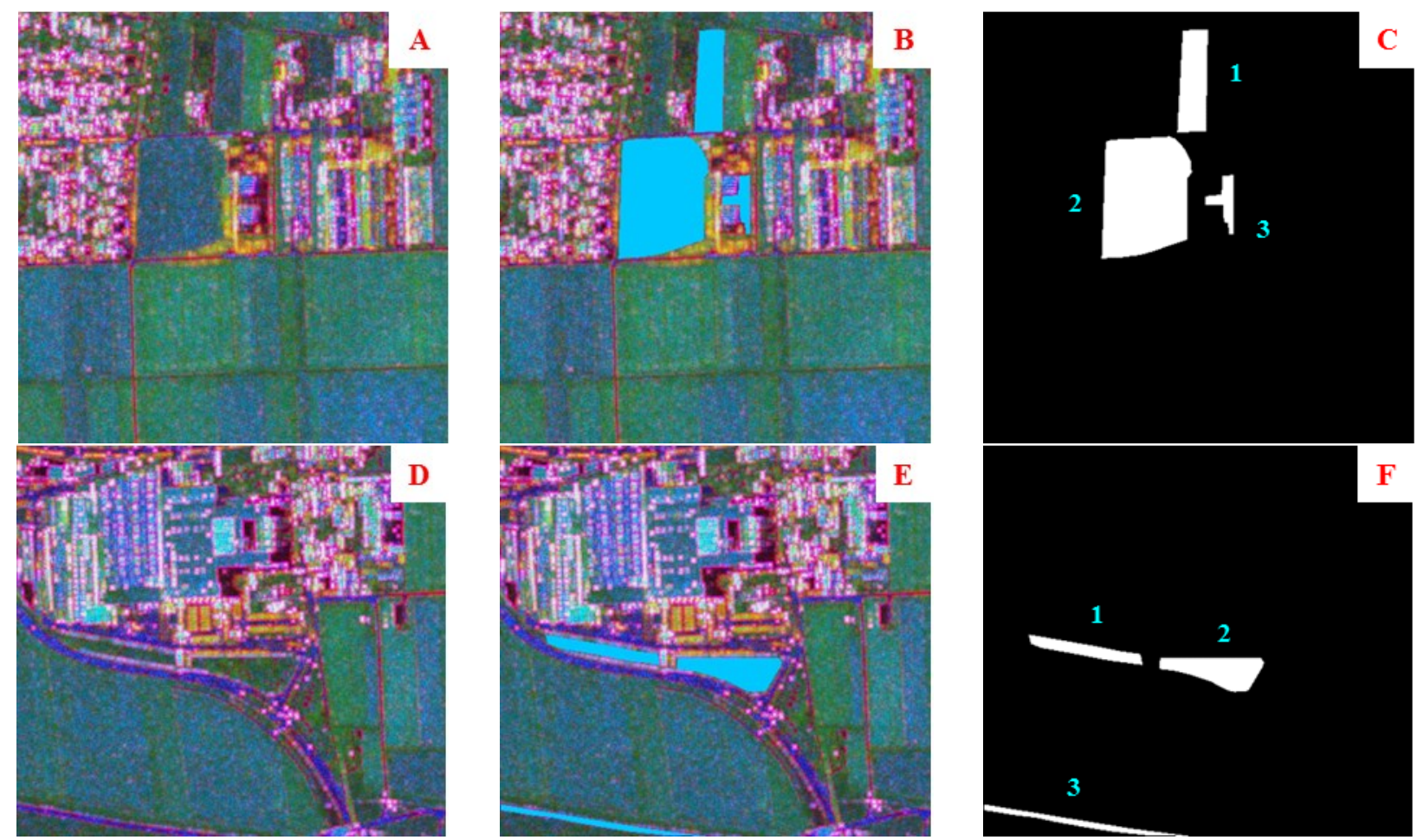

Figure 3: Reference segments. Input CovAmCoh patches (A, D); selected, cyan colored reference segments in CovAmCoh patches (B, E) and reference segments as foreground in binary image patches $(\mathrm{C}, \mathrm{F})$.

\begin{tabular}{|c|c|c|}
\hline & Subset $\mathrm{A}$ & Subset D \\
\hline \multirow{3}{*}{ TP } & Segment 1: $66 \%$ & Segment 1: $80 \%$ \\
\hline & Segment 2: $85 \%$ & Segment 2:84 \% \\
\hline & Segment 3: $60 \%$ & Segment 3: $63 \%$ \\
\hline \multirow{3}{*}{ FN } & Segment 1:31\% & Segment 1: 12\% \\
\hline & Segment 2: $12 \%$ & Segment 2: $13 \%$ \\
\hline & Segment 3:20\% & Segment 3:4 \% \\
\hline \multirow{3}{*}{ FP } & Segment 1:4 \% & Segment 1:8\% \\
\hline & Segment 2:3 \% & Segment 2:4 \% \\
\hline & Segment 3:20\% & Segment 3: $32 \%$ \\
\hline \multirow{3}{*}{ TP } & Segment 1:50\% & Segment 1:84 \% \\
\hline & Segment 2:52\% & Segment 2: $81 \%$ \\
\hline & Segment 3:20\% & Segment 3:27\% \\
\hline \multirow{3}{*}{$\mathrm{FN}$} & Segment 1:4 \% & Segment 1:12\% \\
\hline & Segment 2: $46 \%$ & Segment 2:4 \% \\
\hline & Segment 3: $1 \%$ & Segment $3:<1 \%$ \\
\hline \multirow{3}{*}{ FP } & Segment 1:47\% & Segment 1:4 \% \\
\hline & Segment 2:2 \% & Segment 2: $15 \%$ \\
\hline & Segment 3:79\% & Segment 3:73\% \\
\hline
\end{tabular}

Table 1: Evaluation results. Orange: proposed DAP based segmentation approach; blue: Definiens Developer MRS. Segment numbers and subset letters match to notations given in Figure 3. 